\title{
Proportions of CD4+, CD8+ and B cell subsets are not affected by exposure to HIV or to Cotrimoxazole prophylaxis in Malawian HIV-uninfected but exposed children
}

\author{
Herbert Longwe ${ }^{1,2}$, Kamija S. Phiri², Nyanyiwe M. Mbeye ${ }^{2}$, Thandile Gondwe ${ }^{3}$, Kondwani C. Jambo ${ }^{4}$
} and Wilson L. Mandala $a^{1,4^{*}}$

\begin{abstract}
Background: As a result of successful PMTCT programs, children born from HIV-infected mothers are now effectively protected from contracting the infection. However, it is not well known whether in utero exposure to the virus and the subsequent exposure to Cotrimoxazole (CTX) prophylaxis affect the cell mediated immune system of the children. This observational prospective study was aimed at determining how $C D 4^{+} T, C D 8^{+} T$ and $B$ cell subsets varied in HIV-exposed but uninfected (HEU) children at different ages.

Methods: We recruited HEU and HIV-unexposed and uninfected (HUU) children from 6 months of age and followed them up until they were 18 months old. HEU children received daily CTX prophylaxis beginning at 6 weeks of age until when 12 months of age. Venous blood samples were collected 6 monthly and analysed for different subsets of $C D 8^{+} \mathrm{T}, \mathrm{B}$ cells and totalCD4 ${ }^{+} \mathrm{T}$ cells.

Results: At 6 months of age, HEU children had a lower percentage of total $C D 4^{+} T$ cells compared to HUU children and a lower proportion of naïve $C D 8^{+} T$ cells but higher percentage of effector memory $C D 8^{+} \mathrm{T}$ cells compared to HUU children. HEU and HUU children had similar proportions of all B cell subsets at all ages.

Conclusions: The study showed that the subtle variations in $C D 4^{+}$and $C D 8^{+} T$ cell subsets observed at 6 months do not last beyond 12 months of age, suggesting that HEU children have a robust cell-mediated immune system during first year of life.
\end{abstract}

Trial registration: This article report is not based on results of a controlled health-care intervention

\section{Background}

Lately the number of HIV-exposed but uninfected (HEU) children (HIV negative children born from HIV-infected mothers) has substantially increased due to the scaling up of prevention of mother to child transmission (PMTCT) programs [1]. Although HEU children are not infected with the virus, some studies have still shown that the immune system of these children might have long-term changes either due to in utero exposure to HIV viral particles or

\footnotetext{
* Correspondence: wmandala2002@gmail.com

'Department of Basic Medical Sciences, College of Medicine, University of Malawi, Blantyre, Malawi

${ }^{4}$ Malawi-Liverpool-Wellcome Trust Clinical Research Programme, Blantyre, Malawi

Full list of author information is available at the end of the article
}

antiretroviral (ART) exposure [2]. These changes are thought to have a negative effect on the response to infections as evidenced by the high morbidity in HEU children early in life [3, 4]. However, most of these studies were predominantly undertaken in HEU neonates and during the era before the advent of daily Cotrimoxazole (CTX) prophylaxis for HEU children and that of lifelong ART for pregnant women and lactating mothers.

The adaptive immune system's success in combating infection relies heavily on putting up a robust response and timely and appropriate differentiation of the $\mathrm{B}$ and $\mathrm{T}$ lymphocytes into specific phenotypes. T helper $\left(\mathrm{CD} 4^{+}\right)$ and $\mathrm{T}$ cytotoxic $\left(\mathrm{CD}^{+}\right) \mathrm{T}$ cells are classified either as naïve, effector or memory based on the immune function 
they perform [5]. Naïve $\mathrm{T}$ cells, characterized by the expression of CD45RA ${ }^{+}$, continuously circulate between the secondary lymphoid organs and blood via the lymphatic system [6] and recognize antigens during infection [7]. The naïve T cells are then activated, expand and differentiate into either short-lived effector $\mathrm{T}$ cells or memory precursor effector $\mathrm{T}$ cells that survive for a longer period and provide protection during re-infection with the same pathogen [8]. Memory $\mathrm{T}$ cells are classified either as effector memory $\left(\mathrm{T}_{\mathrm{EM}}\right)\left(\mathrm{CD} 4 \mathrm{RA}^{-} \mathrm{CCR} 7^{-}\right)$or central memory $\left(\mathrm{T}_{\mathrm{CM}}\right) \mathrm{T}$ cells $\left(\mathrm{CD} 45 \mathrm{RA}^{-} \mathrm{CCR} 7^{+}\right)[9]$.

Depending on age, circulating human B cells in healthy individuals comprise naïve $\left(\mathrm{CD} 19^{+} \mathrm{CD} 27^{-} \mathrm{CD} 21^{\mathrm{hi}} \mathrm{CD} 10^{-}\right)$, and memory $\mathrm{B}$ cells that express either unswitched or switched antibody isotypes [10-12]. A population of plasma cells that represent the terminally differentiated mature effector B cells and express $\mathrm{CD} 19^{+} \mathrm{CD} 27^{+} \mathrm{CD} 38^{\mathrm{hi}} \mathrm{CD} 10^{-}$is found in very low numbers $[10,13]$. Protective humoral immune response with optimal longevity depends on generation of memory B cells that are classified into classical memory that are typically $\mathrm{CD} 19^{+} \mathrm{CD} 27^{+} \mathrm{CD} 21{ }^{\text {hi }} \mathrm{CD} 10^{-}$and activated memory expressing $\mathrm{CD} 19^{+} \mathrm{CD} 27^{+} \mathrm{CD} 21^{\mathrm{lo}} \mathrm{CD} 10^{-}$ [14]. Transitional immature $B$ cells, a minor population of circulating B cells, express an immature phenotype (CD19 ${ }^{+} \mathrm{CD} 27^{-} \mathrm{CD} 21^{\text {lo }} \mathrm{CD} 10^{+}$) and have reduced capacity to be activated [10]. Recently a unique memory B cell subpopulation characterized by surface markers $\mathrm{CD} 19^{+} \mathrm{CD} 27$ ${ }^{-} \mathrm{CD} 2{ }^{\mathrm{lo}} \mathrm{CD} 10^{-}$and defined by the expression of the inhibitory receptor Fc-receptor-like-4 (FCRL4) has been identified [15].

Currently little is known about the phenotypic changes of $\mathrm{B}$ and $\mathrm{T}$ cell populations in peripheral blood of HEU children in the era of lifelong ART in pregnancy and lactating mothers and also in HEU children on CTX prophylaxis during the first year of life. Although CTX has been shown to prevent opportunistic bacterial infections in HIV-infected individuals [16-18] little is known of its effect on the development of the immune system in HEU children. Therefore, the aim of this study was to characterize $\mathrm{B}$ and $\mathrm{T}$ cell phenotypic changes in HEU Malawian children during and after completion of daily Cotrimoxazole prophylaxis.

\section{Methods}

\section{Study site, participants and design}

Details of the study site and participants have already been reported previously [19]. Briefly, we recruited HEU children and HUU children from 6 months of age and followed them up until they were 18 months old in Zomba, Malawi. HEU children who had confirmed PCR negative results were randomly selected from the ART clinic at the Zomba central hospital. The children received daily CTX prophylaxis beginning at 6 weeks of age until at 12 months of age where we conducted a rapid test to rule out HIV infection and the mothers were asked to stop breastfeeding. The children were eligible for inclusion if there were otherwise healthy, breastfeeding, not under any medication and if the mothers indicated their intention to reside in the catchment area for the duration of the study. HUU children were those that were HIV-uninfected and recruited from HIV-uninfected mothers residing in the same area as HEU children. Those who were severe malnourished, anaemic, or who presented with other illnesses such as malaria, diarrhea or influenza were excluded from the study. In addition, only those who had not received any malaria medication during the two months before recruitment were included in the HUU group. Both HEU and HUU children were seen every six months and at each visit, $1 \mathrm{ml}$ of venous blood was collected in EDTA tubes for immunophenotyping to characterize the $\mathrm{T}$ and $\mathrm{B}$ cell subsets.

\section{Ethical approval}

The study was reviewed and approved by the College of Medicine Research Ethics Committee (COMREC) (P.05/ 10/954). Individual written informed consent was obtained from the parents or guardians of all the children who participated in the study.

\section{T and B cell characterisation}

Whole blood samples were labelled with anti CD3 PE, anti CD4 PerCP, anti CD8 APC-H7 (all from BD Biosciences, San Jose, California), anti CD45RA FITC (BD Pharmingen, San Jose, California) and anti CCR7 APC (eBiosciences, San Diego, California). The following classification was used for different cell types; $\mathrm{T}$ helper $\left(\mathrm{CD}^{+} \mathrm{CD}^{+}\right)$and $\mathrm{T}$ cytotoxic $\left(\mathrm{CD}^{+} \mathrm{CD}^{+}\right)$. The following additional criteria was used for the different subsets; naïve $\left(C D 45 R A^{+} C C R 7^{+}\right)$, central memory $\left(\mathrm{CD} 45 \mathrm{RA}^{-} \mathrm{CCR} 7^{+}\right)$, effector memory $\left(\mathrm{CD}^{2} 5 \mathrm{RA}^{-} \mathrm{CCR}^{-}\right)$and terminally differentiated effector cells $\left(\mathrm{CD} 45 \mathrm{RA}^{+} \mathrm{CCR}^{-}\right)$.

For the B cells, whole blood was labelled with anti CD19 APC, anti CD21 PE-cy5 (all from BD Pharmingen, San Jose, California), anti CD10 FITC and anti CD27 PE (eBiosciences, San Diego, California). B cells were identified as $\mathrm{CD} 19^{+}$and subpopulations of naïve as $\mathrm{CD} 19^{+} \mathrm{CD} 27^{-} \mathrm{CD} 21^{\mathrm{hi}} \mathrm{CD} 10^{-}$, classical memory as $\mathrm{CD} 19^{+} \mathrm{CD} 27^{+} \mathrm{CD} 21^{\mathrm{hi}} \mathrm{CD} 10^{-}$, activated memory as $\mathrm{CD} 19^{+} \mathrm{CD} 27^{+} \mathrm{CD} 21^{\mathrm{lo}} \mathrm{CD} 10^{-}$, atypical memory $\mathrm{CD} 19^{+}$ $\mathrm{CD} 27^{-} \mathrm{CD} 21^{\mathrm{lo}} \mathrm{CD} 10^{-}$and immature transitional as $\mathrm{CD} 19^{+} \mathrm{CD} 27^{-} \mathrm{CD} 21^{10} \mathrm{CD} 10^{+}$.

\section{Statistical analysis}

Statistical analysis and graphical presentation were done using GraphPad Prism 6 (GraphPad, California, USA) and STATA (StataCorp, Texas, USA). Mann-Whitney $U$ test was used for comparison between the study groups at each visit. Results are presented as medians and interquartile 
Table 1 Comparison of the medians (Interquartile Range) of various cell type subsets at different time points

\begin{tabular}{|c|c|c|c|c|c|c|c|c|c|c|}
\hline \multicolumn{2}{|c|}{ Cell Type or Ratio } & \multicolumn{3}{|l|}{6 Months } & \multicolumn{3}{|l|}{12 Months } & \multicolumn{3}{|l|}{18 Months } \\
\hline & & HEU & HUU & $p$ & HEU & HUU & p & HEU & HUU & $p$ \\
\hline \multicolumn{2}{|c|}{ Total CD4+ T cells } & $55.20(47.50-62.80)$ & $58.40(55.20-67.50)$ & 0.04 & $54.80(42.70-59.30)$ & $51.10(44.60-54.90)$ & 0.62 & $54.80(47.70-58.70)$ & $48.80(39.50-55.30)$ & 0.05 \\
\hline \multicolumn{2}{|c|}{ Effector memory CD4+ T cells } & $6.8(5.13-9.29)$ & $6.47(4.38-8.93)$ & 0.53 & $9.7(6.01-13.2)$ & $7.64(5.30-10.2)$ & 0.19 & $11.9(10.2-14.7)$ & $12.1(9.24-16.5)$ & 0.89 \\
\hline \multicolumn{2}{|l|}{ CD4:CD8 Ratio } & $1.57(1.16-2.18)$ & $1.79(1.47-2.54)$ & 0.08 & $1.50(1.00-1.90)$ & $1.30(1.05-1.78)$ & 0.64 & $1.51(1.10-1.92)$ & $1.18(0.80-1.63)$ & 0.05 \\
\hline \multirow{5}{*}{$\begin{array}{l}\text { CD8+ T cells } \\
\text { Subsets }\end{array}$} & Total CD8+ T & $35.10(29.0-42.3)$ & $32.60(27.40-36.40)$ & 0.15 & $36.40(31.20-43.40)$ & $39.30(31.50-43.40)$ & 0.63 & $36.70(30.60-39.90)$ & $41.70(33.20-47.90)$ & 0.05 \\
\hline & Naiive & $41.40(27.6-51.3)$ & $52.60(36.50-67.50)$ & 0.02 & $46.60(33.50-56.80)$ & $40.50(33.40-64.20)$ & 0.88 & $43.80(36.00-53.30)$ & $38.40(26.70-48.50)$ & 0.17 \\
\hline & Central memory & $1.94(1.37-3.24)$ & $2.11(1.80-3.63)$ & 0.38 & $1.48(0.94-2.68)$ & $1.48(1.01-2.68)$ & 0.82 & $1.17(0.67-1.62)$ & $1.08(0.74-2.16)$ & 0.75 \\
\hline & Effector memory & $19.50(14.90-25.30)$ & $14.30(7.65-22.8)$ & 0.04 & $14.10(8.51-20.0)$ & $13.70(8.68-19.0)$ & 0.89 & $11.20(7.37-15.6)$ & $14.10(8.85-18.0)$ & 0.28 \\
\hline & Terminal Effector & $32.50(21.50-44.80)$ & $27.40(12.1-42.3)$ & 0.15 & $37.40(17.50-49.30)$ & $37.20(20.20-47.80)$ & 0.96 & $41.60(32.60-49.40)$ & $44.70(34.50-57.00)$ & 0.29 \\
\hline \multirow[t]{6}{*}{ B cells Subsets } & Total CD19+ B cells & $23.30(16.10-29.80)$ & $20.7(16.8-29.10)$ & 0.91 & $20.70(13.70-29.10)$ & $20.90(15.20-27.40)$ & 0.98 & $20.10(14.10-25.50)$ & $18.80(12.60-27.30)$ & 0.91 \\
\hline & Naiive & $83.10(79.20-85.60)$ & $83.16(80.70-87.90)$ & 0.35 & $77.15(71.20-78.90)$ & $73.55(70.60-81.90)$ & 0.98 & $70.75(66.40-77.20)$ & $71.53(63.20-74.00)$ & 0.45 \\
\hline & $\begin{array}{l}\text { Immature/ } \\
\text { Transitional }\end{array}$ & $5.14(2.14-7.11)$ & $3.78(2.21-5.09)$ & 0.12 & $2.35(1.39-4.19)$ & $3.38(1.70-4.54)$ & 0.32 & $3.63(2.26-6.98)$ & $4.39(2.41-5.47)$ & 0.91 \\
\hline & Classical Memory & $5.98(5.29-6.73)$ & $5.26(4.59-7.85)$ & 0.58 & $12.11(9.09-13.30)$ & $12.27(8.68-14.4)$ & 0.92 & $12.72(10.2-15.6)$ & $13.05(10.20-15.20)$ & 0.95 \\
\hline & Activated Memory & $1.32(0.77-2.41)$ & $1.37(0.85-2.16)$ & 0.98 & $3.21(2.12-3.85)$ & $2.77(1.64-3.78)$ & 0.36 & $3.17(2.42-4.53)$ & $4.00(3.17-5.22)$ & 0.12 \\
\hline & Atypical Memory & $3.96(2.65-6.17)$ & $4.21(2.75-5.65)$ & 0.91 & $5.99(2.86-7.78)$ & $5.86(3.84-7.77)$ & 0.91 & $6.38(4.28-9.34)$ & $7.53(4.28-11.6)$ & 0.34 \\
\hline
\end{tabular}

The $p$ values presented in bold indicate statistically significant differences between the HEU and HUU values for each cell type 


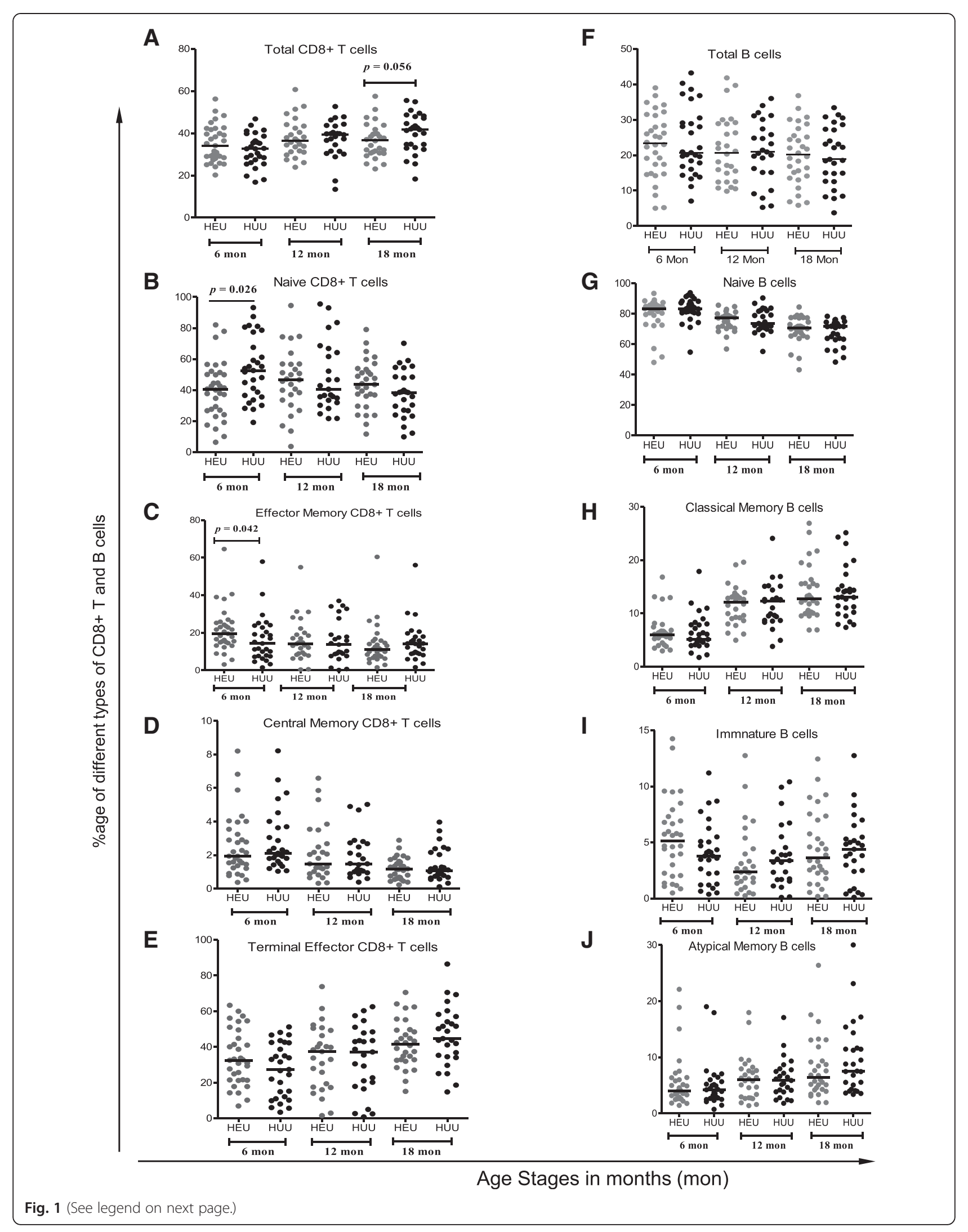


(See figure on previous page.)

Fig. 1 Proportions of different subsets of $\mathrm{CD}^{+} \mathrm{T}$ cells and B cells in HEU and HUU children at different age: Figures a represents percentage of CD $8^{+} T$ cells out of total lymphocytes, $\mathbf{b}$ naïve $C D 8^{+} T$ cells, $\mathbf{c}$ effector memory $C D 8^{+} T$ cells, $\mathbf{d}$ central memory $C D 8^{+} T$ cells and $\mathbf{e}$ terminal effector $\mathrm{CD}^{+}$T cells. Figures $\mathbf{f}$ represents proportion of CD19 ${ }^{+}$B cells as a percentage of total lymphocytes, $\mathbf{g}$ naive B cells, $\mathbf{h}$ classical memory B cell, i immature transitional B cells and $\mathbf{j}$ atypical memory B cells. Horizontal bars represent medians. Comparison between groups was performed with Mann Whitney $U$ test. A difference with a $p$ value of less than 0.05 was considered to be statistically significant

ranges. Any differences between the study groups were considered significant if $p$ values were less than 0.05 .

\section{Results}

Demographic details of the study participants have already been reported previously [19]. At 6 months of age, the median proportion of the total $\mathrm{CD}^{+} \mathrm{T}$ cells in HEU was significantly lower compared to HUU children (55.2 vs. $58.4 \%, p=0.04$ ) (Table 1 ). We did not find any significant differences in the CD4 $\mathrm{T}$ cell subsets between the two groups at all time points (Data not shown). Median proportion of $\mathrm{CD}^{+} \mathrm{T}$ cells was similar between HEU children compared to their HUU counterparts (35.1 vs. $32.6 \%$, $p=0.15$ ) (Fig. 1a). No differences were observed in the median proportions of total $\mathrm{CD}^{+}$and $\mathrm{CD}^{+}$T cells between HEU and HUU children at 12 months: $\mathrm{CD}_{4}^{+}$ (54.8 vs. $51.1 \%, p=0.621), \mathrm{CD}^{+}$(36.4 vs. $39.3 \%, p=$ $0.634)$ and 18 months: $\mathrm{CD}^{+}(54.8$ vs. $48.8 \%, p=0.052)$, $\mathrm{CD}^{+} \mathrm{T}$ cells (36.7 vs. $41.7 \%, p=0.056$ ) (Table 1 ). HEU children had significantly higher ratio of CD4:CD8 $(p=0.05)$ compared to HUU children (Table 1 ).

At 6 months, there was significantly lower proportion of naïve $\mathrm{CD}^{+} \mathrm{T}$ cells (41.4 vs. $\left.52.6 \%, p=0.02\right)$ in HEU children compared to HUU children (Fig. 1b). Interestingly, a significantly high median proportion of $\mathrm{T}_{\mathrm{EM}} \mathrm{CD} 8^{+} \mathrm{T}$ cells (Fig. 1c) was observed in HEU children at 6 months compared to HUU children ( 19.5 vs. $14.3 \%, p=0.04$ ). No significant differences were observed between HEU and HUU children in median proportions of the remaining $\mathrm{CD}^{+}$T subsets (Fig. $1 \mathrm{~b}-\mathrm{e}$ ) at 12 and 18 months of age or effector memory $\left(\mathrm{T}_{\mathrm{EM}}\right) \mathrm{CD} 4+\mathrm{T}$ cells (Table 1 ), either at 6 , 12 or 18 months stages.

Median proportions of $\mathrm{CD} 19^{+} \mathrm{B}$ cells were similar between HEU children compared to their HUU counterparts at 6 months (23.3 vs. $20.7 \%, p=0.91), 12$ months (20.7 vs. $20.9 \%, p=0.98$ ) and 18 months (20.1 vs. $18.8 \%$, $p=0.91)$. No significant differences were observed in the median proportions of all B cell subsets (Fig. $1 \mathrm{~g}-\mathrm{j}$ ), in HEU children compared with HUU children at all three time points.

\section{Discussion}

HEU children have been reported to present with immune abnormalities during infancy, which are thought to be associated with in utero exposure to HIV. We investigated how $\mathrm{B}$ and $\mathrm{T}$ cell phenotypes from peripheral blood of $\mathrm{HEU}$ children vary during and after CTX prophylaxis in comparison with proportions in HUU children. We found that the overall distribution of B and T cell phenotypes was similar among HEU and HUU children at all time points with some few exceptions. The proportion of total $\mathrm{CD} 4^{+} \mathrm{T}$ cells was significantly lower in HEU children at 6 months stage. HEU children had a lower proportion of naïve $\mathrm{CD}^{+}$ $\mathrm{T}$ cells at six months which steadily increased with age. Of much interest was the finding that HEU children had higher proportion of effector memory $\mathrm{CD}^{+} \mathrm{T}$ cells compared to HUU children at 6 months of age.

One study found that HEU Mozambican infants had reduced percentage of $\mathrm{CD}_{4}^{+} \mathrm{T}$ cells at 1 month compared to HUU children [20] and another study showed that total $\mathrm{CD}_{4}^{+} \mathrm{T}$ cell counts were lower in HEU children in early infancy compared to HUU children [21]. Exposure to maternally derived HIV proteins during pregnancy has been suggested to be one possible cause of the reduction in $\mathrm{CD}^{+} \mathrm{T}$ cell counts in HEU children [21-23]. Our findings support the idea that HEU children may have lower proportions of $\mathrm{CD}^{+} \mathrm{T}$ cells during early infancy $[24,25]$, but this abnormality seems not to last for longer than a year.

We also found that the proportions of naïve $\mathrm{CD}^{+} \mathrm{T}$ cells were significantly reduced, whereas that of effector memory $\mathrm{CD}^{+} \mathrm{T}$ cells was significantly higher, in HEU children compared to HUU at 6 months of age. These results support the hypothesis that there may be exposure of the foetal immune system to HIV viral proteins in utero and this may lead to immune activation that drives $\mathrm{CD}^{+} \mathrm{T}$ cells into expansion and differentiation into effector cells [26-28]. However, we expected the in vitro exposure to have equally, if not in a more significantly manner, affected the proportion of effector memory CD4+ $\mathrm{T}$ cells too. The fact that we did not observe this might be explained by the fact that these HIV-infected mothers were on ART during their pregnancy and during the time they were breastfeeding the HEU children.

Alterations in the percentage of B lymphocytes in HIV exposed neonates have been reported before [29] in a study that showed B cells increased in cord blood of HIV exposed neonates, supposedly caused by an increase in $\mathrm{CD} 19^{+} \mathrm{CD}^{+} \mathrm{B}$ cells. Higher $\mathrm{CD} 19^{+} \mathrm{B}$ cell percentages among HEU infants (2 to 6 months) born to mothers with high viral load ( $>1000$ copies $/ \mathrm{ml}$ ) at the time of delivery have also been reported [26]. The current study showed no evidence of $\mathrm{CD} 19^{+} \mathrm{B}$ cell subset alteration in 
HEU children at 6,12 and 18 months of age. This may imply that the B cells ability to produce antibodies may not be impaired in HEU children.

The study had a number of limitations. Firstly, no data on lymphocyte subset proportions at birth for the children were collected, as this would have provided an ideal baseline for comparison with the subsequent follow-up stages. Secondly, although all the HIV-infected mothers were confirmed to be on ART, no data on CD4 T cell counts or HIV viral load levels, or duration on ART were collected from the mothers at the time of giving birth and during the follow up period of the study. Maternal disease status, especially their viral load at the time of recruitment, might have affected the extent of infant HIV exposure in utero and through breast milk [26]. As such it is not easy for the study to ascertain whether the lack of differences in the $\mathrm{B}$ and $\mathrm{T}$ cell phenotypes observed in the HEU group was due to reduced exposure to HIV viral particles or not.

Having a control group comprising HEU children who were not on CTX prophylaxis would have allowed us to clearly specify if what was observed in the HEU on CTX prophylaxis was indeed an effect of CTX on the proportion of various cell subsets and not due to in utero HIV exposure. However, ethically it was not possible to have such a group since the current Malawi Government guidelines clearly state that every HEU child should be on CTX for the first year of life for the obvious beneficial effects. We therefore used HUU recruited from same communities as controls instead.

\section{Conclusions}

In conclusion, we have shown that there are no long term alterations in the proportion of $\mathrm{B}$ and $\mathrm{T}$ cellsubsets in HEU children compared to HUU children suggesting that HEU children have a robust cellmediatedimmune system during the first year of life.

\section{Abbreviations}

ART: Antiretroviral therapy; CTX: Cotrimoxazole; HEU: HIV-exposed but uninfected; HUU: HIV-unexposed and uninfected; PMTCT: Prevention of mother to child transmission.

\section{Competing interests}

The authors declare that they have no competing interests.

\section{Authors' contributions \\ Conceived and designed the experiments: KSP, HL, WLM. Performed the experiments: $\mathrm{HL}$ and KJ. Analyzed the data: HL KCJ WLM. Wrote the paper: HL WLM, KCJ. Coordination and clinical work: NM and TG. All authors amended the draft and approved the final manuscript.}

\section{Acknowledgements}

We are grateful to all the parents and guardians for giving their consent and the children for their cooperation. We are also grateful to Mr. Maurice Mutisya and Dr. Simbarashe Takuva for offering statistical help. We thank the whole study team in Zomba for collecting the data.

\section{Funding}

The study was funded by the Malawi Health Research Capacity Strengthening Initiative (HRCSI), a DFID and Wellcome Trust funded programme and the HIV Research Trust UK. This research was also partially funded by Fellowship awards provided by the Consortium for Advanced Research Training in Africa (CARTA) andSouthern Africa Consortium for Research Excellence (SACORE). CARTA has been funded by the Wellcome Trust (UK) (Grant No: 087547/Z/08/Z), the Department for International Development (DfID) under the Development Partnerships in Higher Education (DelPHE), the Carnegie Corporation of New York (Grant No: B 8606), the Ford Foundation (Grant No: 1100-0399), Google.org (Grant No: 191994), Sida (Grant No: 54100029) and the Bill and Melinda Gates Foundation (Grant No: 51228).

\section{Author details}

${ }^{1}$ Department of Basic Medical Sciences, College of Medicine, University of Malawi, Blantyre, Malawi. ${ }^{2}$ Tropical Haematology Research Unit, College of Medicine, University of Malawi, Blantyre, Malawi. ${ }^{3}$ Department of Public Health, College of Medicine, University of Malawi, Blantyre, Malawi. ${ }^{4}$ Malawi-Liverpool-Wellcome Trust Clinical Research Programme, Blantyre, Malawi.

Received: 29 May 2015 Accepted: 25 August 2015

Published online: 28 August 2015

\section{References}

1. Study EC. Mother-to-child transmission of HIV infection in the era of highly active antiretroviral therapy. Clin Infect Dis. 2005;40(3):458-65.

2. Afran L, Garcia Knight M, Nduati E, Urban BC, Heyderman RS, Rowland-Jones SL. HIV-exposed uninfected children: a growing population with a vulnerable immune system? Clin Exp Immunol. 2014;176(1):11-22.

3. Slogrove A, Reikie B, Naidoo S, De Beer C, Ho K, Cotton M, et al. HIV-exposed uninfected infants are at increased risk for severe infections in the first year of life. J Trop Pediatr. 2012;58(6):505-8.

4. Marinda E, Humphrey JH, Iliff PJ, Mutasa K, Nathoo KJ, Piwoz EG, et al. Child mortality according to maternal and infant HIV status in Zimbabwe. Pediatr Infect Dis J. 2007;26(6):519-26.

5. Janeway C. T cell mediated immunity. In: Murphy K, Travers P, Walport M, editors. Janeway's Immunobiology. 7th ed. New York, US: Garland Science; 2008.

6. Lewis M, Tarlton JF, Cose S. Memory versus naive T-cell migration. Immunol Cell Biol. 2008;86(3):226-31.

7. Yamane H, Paul WE. Early signaling events that underlie fate decisions of naive CD4(+) T cells toward distinct T-helper cell subsets. Immunol Rev. 2013;252(1):12-23.

8. Rohr JC, Gerlach C, Kok L, Schumacher TN. Single cell behavior in T cell differentiation. Trends Immunol. 2014;35(4):170-7.

9. Sallusto F, Geginat J, Lanzavecchia A. Central memory and effector memory $T$ cell subsets: function, generation, and maintenance. Annu Rev Immunol. 2004;22:745-63.

10. Caraux A, Klein B, Paiva B, Bret C, Schmitz A, Fuhler GM, et al. Circulating human $B$ and plasma cells. Age-associated changes in counts and detailed characterization of circulating normal CD138- and CD138+ plasma cells. Haematologica. 2010;95(6):1016-20.

11. Mandala WL, MacLennan JM, Gondwe EN, Ward SA, Molyneux ME, MacLennan CA. Lymphocyte subsets in healthy Malawians: implications for immunologic assessment of HIV infection in Africa. J Allergy Clin Immunol. 2010;125(1):203-8.

12. Moir S, Fauci AS. B cells in HIV infection and disease. Nat Rev Immunol. 2009;9(4):235-45.

13. Robillard N, Wuilleme S, Moreau P, Bene MC. Immunophenotype of normal and myelomatous plasma-cell subsets. Front Immunol. 2014;5:137.

14. Weiss GE, Crompton PD, Li S, Walsh LA, Moir S, Traore B, et al. Atypical memory $B$ cells are greatly expanded in individuals living in a malaria-endemic area. J Immunol. 2009;183(3):2176-82.

15. Moir S, Ho J, Malaspina A, Wang W, DiPoto AC, O'Shea MA, et al. Evidence for HIV-associated B cell exhaustion in a dysfunctional memory B cell compartment in HIV-infected viremic individuals. J Exp Med. 2008;205(8):1797-805.

16. Anglaret $X$, Chene $G$, Attia A, Toure $S$, Lafont $S$, Combe P, et al. Early chemoprophylaxis with trimethoprim-sulphamethoxazole for HIV-1-infected 
adults in Abidjan, cote d'Ivoire: a randomised trial. Cotrimo-Cl study group. Lancet. 1999;353(9163):1463-8.

17. Mermin J, Lule J, Ekwaru JP, Malamba S, Downing R, Ransom R, et al. Effect of co-trimoxazole prophylaxis on morbidity, mortality, CD4-cell count, and viral load in HIV infection in rural Uganda. Lancet. 2004;364(9443):1428-34.

18. Walker AS, Mulenga V, Ford D, Kabamba D, Sinyinza F, Kankasa C, et al. The impact of daily cotrimoxazole prophylaxis and antiretroviral therapy on mortality and hospital admissions in HIV-infected Zambian children. Clin Infect Dis. 2007;44(10):1361-7.

19. Longwe H, Jambo KC, Phiri KS, Mbeye N, Gondwe T, Hall T, et al. The effect of daily co-trimoxazole prophylaxis on natural development of antibody-mediated immunity against $P$. falciparum malaria infection in HIV-exposed uninfected Malawian children. PLoS One. 2015;10(3):e0121643.

20. Moraleda C, de Deus N, Serna-Bolea C, Renom M, Quinto L, Macete E, et al. Impact of HIV exposure on health outcomes in HIV-negative infants born to HIV-positive mothers in Sub-Saharan Africa. J Acquir Immune Defic Syndr. 2014;65(2):182-9.

21. Clerici M, Saresella M, Colombo F, Fossati S, Sala N, Bricalli D, et al. T-lymphocyte maturation abnormalities in uninfected newborns and children with vertical exposure to HIV. Blood. 2000;96(12):3866-71.

22. Ono E, Santos AM N d, de Menezes Succi RC, Machado DM, de Angelis DS, Salomao R, et al. Imbalance of naive and memory T lymphocytes with sustained high cellular activation during the first year of life from uninfected children born to HIV-1-infected mothers on HAART. Braz J Med Biol Res. 2008;41(8):700-8.

23. Nielsen SD, Jeppesen DL, Kolte L, Clark DR, Sorensen TU, Dreves AM, et al. Impaired progenitor cell function in HIV-negative infants of HIV-positive mothers results in decreased thymic output and low CD4 counts. Blood. 2001;98(2):398-404.

24. Bunders M, Thorne C, Newell ML. Maternal and infant factors and lymphocyte, CD4 and CD8 cell counts in uninfected children of HIV-1-infected mothers. AIDS. 2005;19(10):1071-9.

25. Gesner M, Papaevangelou V, Kim M, Chen SH, Moore T, Krasinski K, et al. Alteration in the proportion of CD4 T lymphocytes in a subgroup of human immunodeficiency virus-exposed-uninfected children. Pediatrics. 1994;93(4):624-30.

26. Kakkar F, Lamarre V, Ducruet T, Boucher M, Valois S, Soudeyns H, et al. Impact of maternal HIV-1 viremia on lymphocyte subsets among HIV-exposed uninfected infants: protective mechanism or immunodeficiency. BMC Infect Dis. 2014;14(1):236.

27. Kidzeru EB, Hesseling AC, Passmore JA, Myer L, Gamieldien H, Tchakoute CT, et al. In-utero exposure to maternal HIV infection alters T-cell immune responses to vaccination in HIV-uninfected infants. AIDS. 2014;28(10):1421-30.

28. Kuhn L, Coutsoudis A, Moodley D, Trabattoni D, Mngqundaniso N, Shearer GM, et al. T-helper cell responses to HIV envelope peptides in cord blood: protection against intrapartum and breast-feeding transmission. AIDS. 2001;15(1):1-9.

29. Borges-Almeida E, Milanez HM, Vilela MM, Cunha FG, Abramczuk BM, Reis-Alves SC, et al. The impact of maternal HIV infection on cord blood lymphocyte subsets and cytokine profile in exposed non-infected newborns. BMC Infect Dis. 2011;11:38

\section{Submit your next manuscript to BioMed Central and take full advantage of:}

- Convenient online submission

- Thorough peer review

- No space constraints or color figure charges

- Immediate publication on acceptance

- Inclusion in PubMed, CAS, Scopus and Google Scholar

- Research which is freely available for redistribution 